\title{
Acousto-Optical Tunable Transmissive Grating Beam Splitter
}

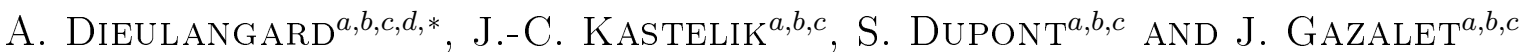 \\ ${ }^{a}$ Université Lille Nord de France, 59000 Lille, France \\ ${ }^{b}$ Université de Valenciennes et du Hainaut-Cambrésis, IEMN-DOAE, Le Mont Houy, \\ 59313 Valenciennes Cedex 9, France \\ ${ }^{c}$ CNRS, UMR 8520, 59650 Villeneuve d'Ascq, France \\ ${ }^{d}$ AA Opto-electronic SA, 18, rue Nicolas Appert, 91898 Orsay, France
}

\begin{abstract}
We present a tunable transmissive grating beam splitter for multiple laser line separation based on acoustooptic interaction in tellurium dioxide. Acousto-optic devices are well known for light modulation, frequency shifting, filtration or deflection. Unlike the classical operating modes of acousto-optic devices, we consider the simultaneous diffraction of several optical wavelengths by a single ultrasonic frequency. The device is based on the Bragg anisotropic interaction in the specific "tangent phase matching" configuration. The acousto-optic interaction takes place with a single ultrasonic frequency and the diffraction efficiency remains high over a wide optical spectral range. The different diffracted beams are then angularly well separated, due to the slow velocity of the ultrasonic wave propagating in tellurium dioxide. The optical bandwidth is directly related to the operating ultrasonic frequency. Numerical calculations were carried out to determine the main parameters of the device: operating ultrasonic frequency, optical bandwidth, tunability range, crystalline cut and transducer length. A practical device has been designed for visible spectrum. Experimental results are presented as for example a spectral bandwidth from $450 \mathrm{~nm}$ to $550 \mathrm{~nm}$ with a carrier frequency $f_{0}=125 \mathrm{MHz}$.
\end{abstract}

DOI: $10.12693 /$ APhysPolA.127.66

PACS: 78.20.hb, 42.79.Jq, 43.35.Sx

\section{Introduction}

Acousto-optic devices are based on the diffraction of optical beams by ultrasonic waves. These interactions take place in specific crystals exhibiting good photoelastic properties. Among available acousto-optic materials, tellurium dioxide is truly remarkable due to its highly optical and acoustical anisotropic properties leading to specific configurations of acousto-optic interaction [1-3]. Acousto-optic devices are mainly used for light modulation, frequency shifting, deflection, or optical filtering [4]. In this paper, we present the investigation process leading to a dynamic acousto-optic grating beam splitter, based on the well know tangent phase matching configuration (TPM) widely used in acousto-optic deflector [5]. The TPM configuration gives a larger spectral bandwidth at the condition that both the incident optical angle and the ultrasonic frequency are well selected, as shown in this paper.

The device can be considered as a dynamic transmissive diffraction grating [6] and the operating principle is depicted in Fig. 1. A single slow ultrasonic wave propagating in a tellurium dioxide crystal interacts simultaneously with all the optical wavelengths of the incident beam. The different diffracted beams are then angularly well separated, due to the low velocity of the ultrasonic wave. The proposed device operates with linear polarized incident beams coupled to the extraordinary mode

*corresponding author; e-mail: anthony.dieulangard@gmail.com of the tellurium dioxide crystal. In order to maximize the diffraction efficiency on a large spectral bandwidth, computational simulations have been performed leading to a specific design. Experimental results show that the diffraction efficiency is higher than $60 \%$ in the spectral range $450-550 \mathrm{~nm}$. In the next section, we present the basic concept of the grating beam splitter based on the wave vector diagrams in a multi-wavelength anisotropic interaction. The next section is then devoted to the numerical calculations and the experimental measurements obtained with a practical device.

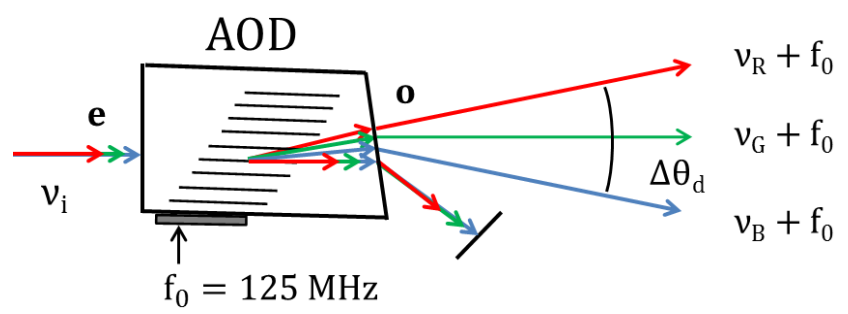

Fig. 1. Operating principle of the acousto-optical grating beam splitter.

\section{Wave vector diagram}

The "tangent phase matching" acousto-optic configuration in tellurium dioxide has been extensively detailed in a recent paper [7] considering wave vector diagram, phase mismatch, crystalline cut, bandwidth etc. In order to illustrate the configuration of multi-wavelength anisotropic interaction, we consider the simultaneous 
wave vector diagrams at different wavelengths in the visible range with a single ultrasonic shear wave propagating with an angular tilt from the [110] axis frequency (Fig. 2). As for example, the phase matched interaction (phase mismatch $\Delta \Phi=0$ ) is established for the blue wavelength $\lambda_{\mathrm{B}}$ with the ultrasonic frequency $f_{0}$ (wave vector $\boldsymbol{K}$ ) and the incident angle $\theta_{\mathrm{i}}$. For the green wavelength $\lambda_{\mathrm{G}}$, with the same ultrasonic frequency $f_{0}$ and incident angle $\theta_{\mathrm{i}}$, the interaction takes place with a given phase mismatch. However, another phase matched interaction is obtained for the orange wavelength $\lambda_{\mathrm{OR}}$. Finally, the phase mismatch also increases for red light $\lambda_{\mathrm{R}}$. The diffraction efficiency rapidly decreases for higher optical wavelengths and the same behavior occurs for lower optical wavelengths as for ultraviolet light $\lambda_{\mathrm{UV}}$. Thus, we obtain a spectral bandwidth with ripple depth criteria as those usually found for the frequency bandwidth of acousto-optic deflectors operating with a single optical wavelength. A precise selection of the couple (incident angle $\theta_{i}$, ultrasonic frequency $f_{0}$ ) leads to classical criteria of $-3 \mathrm{~dB}$ or $-0.5 \mathrm{~dB}$ bandwidth.

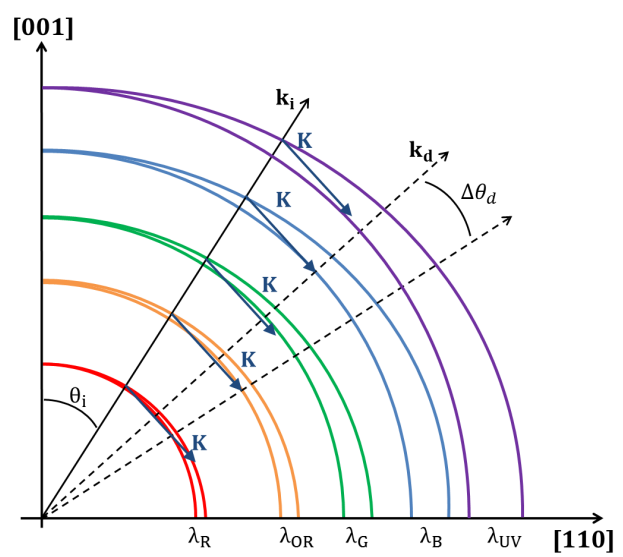

Fig. 2. Wave-vector diagram of anisotropic interaction in the tangential phase matching configuration for several optical wavelengths and a single ultrasonic frequency.

\section{Numerical calculations and experimental validation}

The experimental setup includes several laser wavelengths issued from an argon laser (458, 476, 488, 496, and $514 \mathrm{~nm}$ ) combined with a $532 \mathrm{~nm}$ laser source. The radio-frequency generator operates in a range starting from 70 to $140 \mathrm{MHz}$. For clarity, Fig. 3a shows the calculated diffraction efficiencies for three optical wavelengths 458,496 , and $532 \mathrm{~nm}$ and for $\theta_{\mathrm{i}}=5.48^{\circ}$. For $\lambda=458 \mathrm{~nm}$ a phase matched interaction occurs for a single ultrasonic frequency $(123.7 \mathrm{MHz})$. For the wavelength $\lambda=496 \mathrm{~nm}$, two phase matched interactions are possible (frequencies $95.5 \mathrm{MHz}$ and $129 \mathrm{MHz}$ ) as well as for $\lambda=532 \mathrm{~nm}$ (frequencies $81.5 \mathrm{MHz}$ and $125 \mathrm{MHz}$ ). For experimental validation a single ultrasonic frequency has been fixed $f_{0}=125 \mathrm{MHz}$. A practical tellurium dioxide acousto-optic device, based on usual AOD configuration, has been specially built for the experiment by AA Opto-electronic. The crystalline cut, corresponding to the angular tilt of the slow shear wave from the [110] axis is $7.5^{\circ}$. The transducer length $W=5.5 \mathrm{~mm}$ leads to a frequency bandwidth from 70 to $140 \mathrm{MHz}$ in the TPM configuration. Figure $3 \mathrm{~b}$ shows the experimental measurements of the diffraction efficiencies for the three wavelengths and a good agreement is obtained.

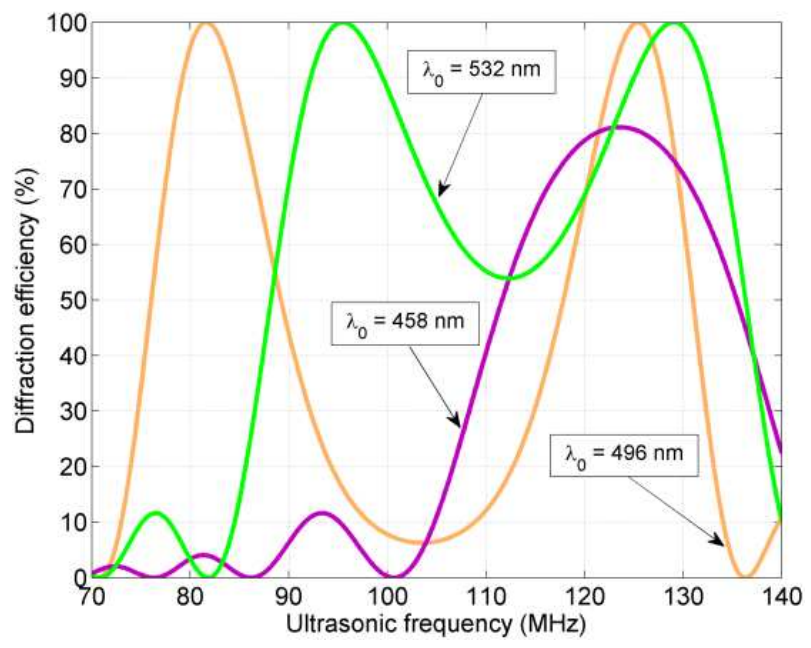

Fig. 3. Frequency bandwidth of the acousto-optic grating beam splitter for three optical wavelengths: 458, 496, and $532 \mathrm{~nm}$. (a) Numerical calculations; (b) experimental measurements.

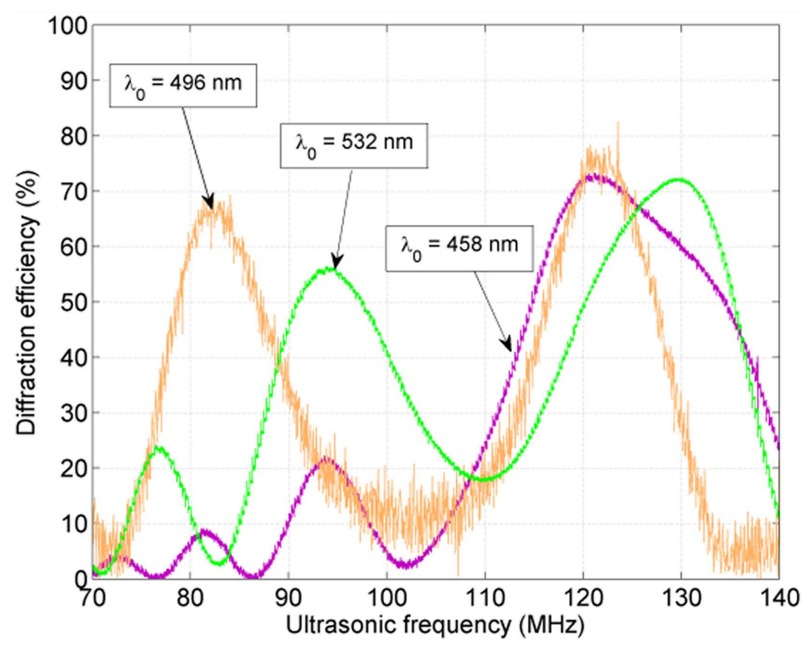

Fig. 4. Photograph of the blue-green diffracted light beams.

All the wavelengths have been tested simultaneously and the photograph reported in Fig. 4 shows the corresponding diffracted beams. As expected a large angular separation between the diffracted beams is obtained, due to the low velocity of the ultrasonic shear wave 
$\left(676 \mathrm{~ms}^{-1}\right)$. The separation angle between the diffracted beam corresponding to $\lambda=458 \mathrm{~nm}$ and the one corresponding to $\lambda=532 \mathrm{~nm}$ is $1^{\circ}$. The measured diffraction efficiency is higher than $60 \%$ for all the wavelengths.

\section{Conclusion}

In this paper, we have presented an original configuration for acousto-optic devices. It offers a new application for acousto-optic deflectors, considering polychromatic optical incident beam and single ultrasonic frequency. This leads to a wideband tunable transmissive grating beam splitter. The system is based on the Bragg anisotropic acousto-optic interactions in tellurium dioxide crystal. Acousto-optic interactions are based on the well-known tangential phase matching configuration. Usually used for angular deflection of a monochromatic beam tuning of the ultrasonic frequency, it typically leads to a wide frequency bandwidth, taking the advantage of a low phase mismatch on a large frequency domain.

This paper shows that a similar behavior is predictable in the spectral domain, according to a careful selection of the incident angle and the ultrasonic frequency. In the same way as for the frequency bandwidth of an AOD, the spectral bandwidth can be fitted and selective ripple criteria can be chosen setting optical beam incidence. The device has been designed for a spectral bandwidth starting from 450 to $550 \mathrm{~nm}$ with a $-0.5 \mathrm{~dB}$ ripple depth. Larger spectral bandwidths can be achieved with bigger ripple depth criteria. Acousto-optic deflectors used as dispersive elements present a wider angular separation in comparison with conventional prisms or grating dispersive elements. Moreover, the Bragg acousto-optic interactions imply only one diffraction order, leading to high diffraction efficiency. It is a dynamic diffraction grating: the intensity of the diffracted orders can be modulated. The proposed configuration can be extended to any optical domain in the transparency range of tellurium dioxide which is large from ultraviolet to infrared light beams.

\section{References}

[1] V. Balakshy, A. Voloshin, Opt. Spectrosc. 110, 788 (2011).

[2] V.B. Voloshinov, N.V. Polikarpova, O.Y. Makarov, N.P. Solodovnikov, V.N. Ginzburg, E.V. Katin, E.A. Khazanov, V.V. Lozhkarev, I.V. Yakovlev, Appl. Opt. 48, C55 (2009).

[3] V.Y. Molchanov, S.I. Chizhikov, Appl. Opt. 48, C118 (2009).

[4] N. Savage, Nature Photon. 4, 728 (2010).

[5] P. Maak, L. Jakab, A. Barocsi, P. Richter, Opt. Commun. 172, 297 (1999).

[6] D.R. Suhre, L.H. Taylor, N.B. Singh, W.R. Rosch, Proc. SPIE 3584, 142 (1999).

[7] J.-C. Kastelik, S. Dupont, K.B. Yushkov, Ultrasonics 5, 219 (2013). 